\title{
Characterization of Dynamic Material Properties of Light Alloys for Crashworthiness Applications
}

\author{
Nuno Peixinho*, Claudia Doellinger \\ Departamento de Engenharia Mecânica, University of Minho - UMinho, \\ Campus de Azurém, CEP 4800-058, Guimarães, Portugal
}

Received: June 14, 2010; Revised: September 30, 2010

\begin{abstract}
This paper presents results on the tensile testing of AZ31B-H24 magnesium alloy and 6111-T4 aluminium alloy at different strain rates. These materials are strong candidates for use in crashworthy automotive components and parts due to their well-balanced combination of strength, stiffness and density. To test their application in the auto industry an understanding of material behaviour at relevant strain rates is needed, as well as constitutive equations suitable for use in analytical and numerical calculations. Mechanical properties were determined from tensile tests using flat sheet samples, employing two different test techniques: a servo-hydraulic machine and a tensile-loading Hopkinson bar. The test results were used to compare different mechanical properties of the tested materials and to validate constitutive equations intended to provide a mathematical description of strain rate dependence. The Cowper-Symonds equation was examined.
\end{abstract}

Keywords: light alloys, material properties, crashworthiness, constitutive models

\section{Introduction}

The improvements demanded in fuel efficiency and $\mathrm{CO}_{2}$ emissions in the auto industry have resulted in a renewed interest in light alloys. This trend is set to continue with stricter emissions legislation and changing public perception. There has been a dramatic increase in the use of aluminium alloys in recent years: between 1995 and 2000, their use in automotive components increased by over $80 \%^{[1]}$. Weight reductions of up to $40 \%$ have been reported as possible in aluminium intensive space frames ${ }^{1}$. Magnesium alloys are the lightest metallic structural materials and as such have great potential for use in the automotive industry. The use of magnesium alloys in car parts is predicted to grow from the $3 \mathrm{~kg}$ per car used in 2005 to $20 \mathrm{~kg}$ in 2010 and $50 \mathrm{~kg}$ by 2015 as legislation on emissions and pressure for weight reduction gain more importance ${ }^{2}$.

When considering the use of light alloys in crashworthy components, the dynamic behaviour of the materials used must be considered. Furthermore, high strain-rates are also a feature in some manufacturing processes, such as electromagnetic forming ${ }^{3}$. Knowledge of material properties at different strain-rates and the development of constitutive equations capable of providing a reliable description of that behaviour, is therefore, highly relevant. One of the most commonly used constitutive equations for steel grades is the Cowper-Symonds equation and its variations $s^{4,5}$, being extensively used in the car industry for the numerical simulation of crashworthiness events. There is a gap in the literature on the constitutive parameters of this equation for magnesium and aluminium alloys, which could be of use for the numerical simulation of crashworthiness events.

Aluminium is commonly considered to have low strain rate sensitivity, particularly for alloys of higher strength. Several studies have been conducted that present dynamic material properties for different alloys, heat treatment and/or processing conditions ${ }^{6,7,8}$. For magnesium alloys a higher sensitivity to strain rate is present, being dependent on the processing (cast or wrought) and alloying used. Li et al. ${ }^{9}$ presented results of compression tests of alloy AZ31B at different strain rates and temperatures. Valle and Ruano ${ }^{10}$ analyzed the influence of grain size on the strain rate sensitivity of a AZ31 magnesium alloy and concluded that strain rate sensitivity increases strongly for decreasing grain size below $15 \mu \mathrm{m}$. The strain rate sensitivity of magnesium alloys must be considered in numerical simulation of impact events in order to obtain good correlation with experiments, as presented in a study on denting of magnesium and aluminium panels ${ }^{11}$.

\section{Materials, Samples and Experimental Techniques}

The tested materials were: magnesium alloy AZ31B-H24 (sheet thickness of $0.9 \mathrm{~mm}$ ) and aluminium alloy 6111-T4 (thickness $1.09 \mathrm{~mm}$ ). Table 1 presents the chemical composition that was obtained in a spectrometer Phillips X'Unique II. Quasi-static mechanical properties were obtained following EN 10002-1 standard using a universal tensile testing machine (DARTEC, $600 \mathrm{KN}$ ), including the tensile yield strength at $0,2 \%$ offset $\left(R_{p 0.2}\right)$, Ultimate Tensile Strength (UTS) and elongation to fracture for an initial gauge length of $80 \mathrm{~mm}$ are presented in Table 2 .

Samples used in tensile testing were cut in the rolling direction. The quasi-static and dynamic tensile tests were performed using distinct techniques:

- Servo-hydraulic testing machine (DARTEC, $600 \mathrm{kN}$ capacity), used for lower loading speeds $(0.1 \mathrm{~mm} / \mathrm{s})$, here referred to as quasi-static loading and for tests at an intermediate loading speed of $90 \mathrm{~mm} / \mathrm{s}$. In the quasi-static tests, values of engineering stress and strain were determined using the strain gauge load cell of the test machine (DARTEC, M1000 RD, $600 \mathrm{kN}$ ), and a strain-gauge extensometer clip-gauge (SANDNER model EXA 100-10), with a gauge length of $100 \mathrm{~mm}$. Elongation to fracture was measured using a reference length of $80 \mathrm{~mm}$. Tests at intermediate loading speed $(90 \mathrm{~mm} / \mathrm{s})$ used smaller samples with a parallel length of $40 \mathrm{~mm}$ and reference gauge length of $20 \mathrm{~mm}$. This geometry was used in order to obtain higher strain-rates (approx. 2.25/s). This nominal strain rate was calculated by dividing the linear speed of the testing machine by the parallel length of the sample. The tests at intermediate loading speed used strain gauges attached to the main body of the sample to obtain strain readings in the initial stage of 
Table 1. Chemical composition.

\begin{tabular}{lrrrllllllllll}
\hline & $\mathrm{Mg} \%$ & $\mathrm{Al} \%$ & $\mathrm{Si} \%$ & $\mathrm{P} \%$ & $\mathrm{~S} \%$ & $\mathrm{Cl} \%$ & $\mathrm{~K} \%$ & $\mathrm{Ca} \%$ & $\mathrm{Ti} \%$ & $\mathrm{Mn} \%$ & $\mathrm{Fe} \%$ & $\mathrm{Ni} \%$ & $\mathrm{Zn} \%$ \\
\hline AA6111-T4 & 0.76 & 97.45 & 0.99 & 0.013 & - & 0.039 & 0.021 & 0.040 & 0.054 & 0.14 & 0.24 & 0.006 & 0.014 \\
AZ31B-H24 & 91 & 4.29 & 0.29 & 0.07 & 0.28 & 0.32 & 0.103 & 0.19 & 0.011 & 0.95 & 0.113 & 0.003 & 2.4 \\
\hline
\end{tabular}

Table 2. Mechanical properties.

\begin{tabular}{cccc}
\hline & $R_{p 0.2}[\mathrm{MPa}]$ & UTS [MPa] & Elongation, \% \\
\hline AA6111-T4 & 149.2 & 240.1 & 23.2 \\
AZ31B-H24 & 197.4 & 261.2 & 7.7 \\
\hline
\end{tabular}

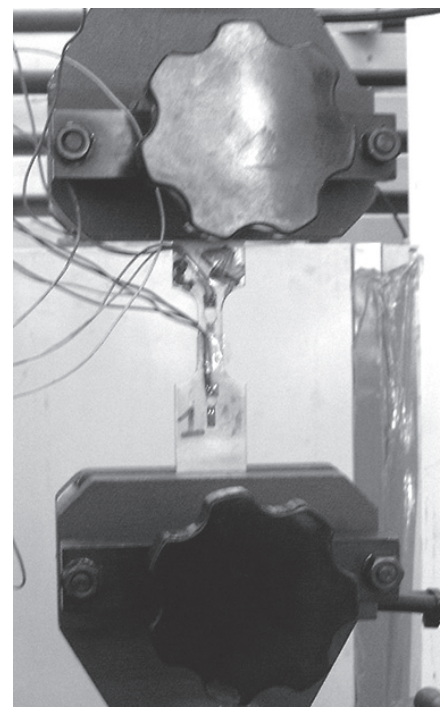

Figure 1. Specimen used in tests at $2.25 / \mathrm{s}$.

the tensile test (Figure 1). Strain gauges were also placed at the top edge of the sample. Since this region remains elastic these gauges were used to confirm the load at this higher speed, hence avoiding fluctuations observed in the load-cell signals. Readings from the strain gauges were recorded using HBM Spyder- 8 equipment and analysed on a personal computer.

- Tensile loading Hopkinson pressure bar, used for obtaining strain rates of approximately 1000/s (Figure 2). With this equipment a striker tube is fired from a gas gun and impacts an anvil. A tensile pulse is generated in the incident bar from the impact and propagates into the sample. The grip is appropriately designed for plate type samples. Part of the incident pulse is transmitted into the sample and propagates through the transmitted bar as a tensile pulse. The rest of the pulse reflects into the incident bar as a compressive pulse. The transmitted and reflected pulses are measured with strain gauges placed at the incident and transmitted bars. Output signals from the equilibrated Wheatstone circuits were amplified using Fylde FE-527-SGA dynamic strain amplifiers. Readings were recorded using an oscilloscope Yokogawa model DL1540L and subsequently analysed on a personal computer. From the strain gauge readings, stress-strain curves were obtained following the procedure indicated in Kang et al. ${ }^{12}$. Additionally, local strain was measured using strain gauges placed on the main body of the specimen. This was useful in order to confirm results obtained with the Hopkinson bar procedure in the initial phase of the tests.

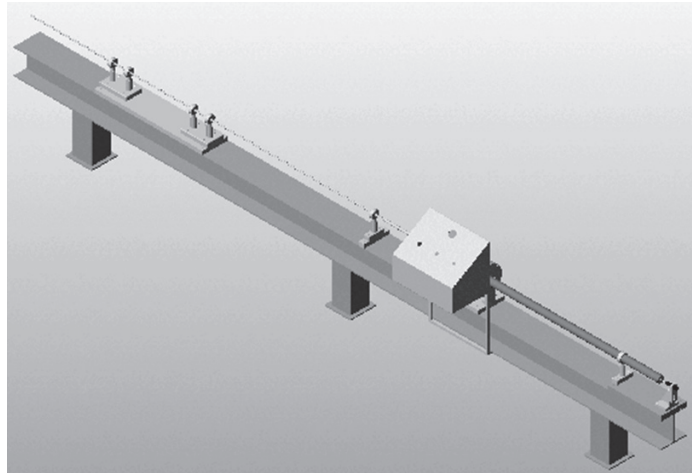

(a)

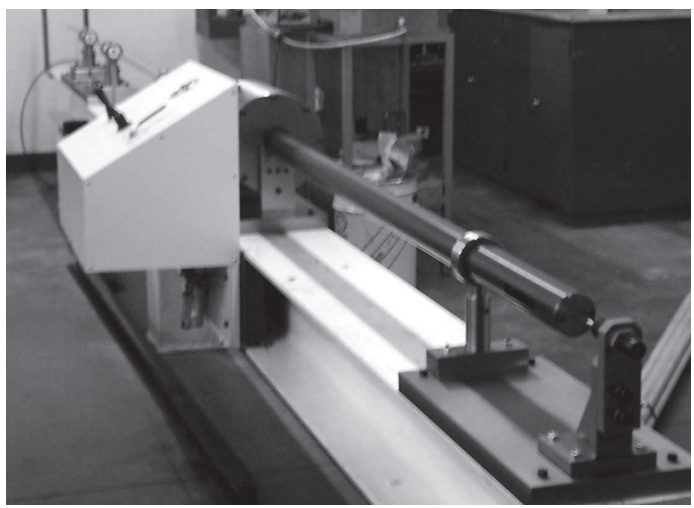

(b)

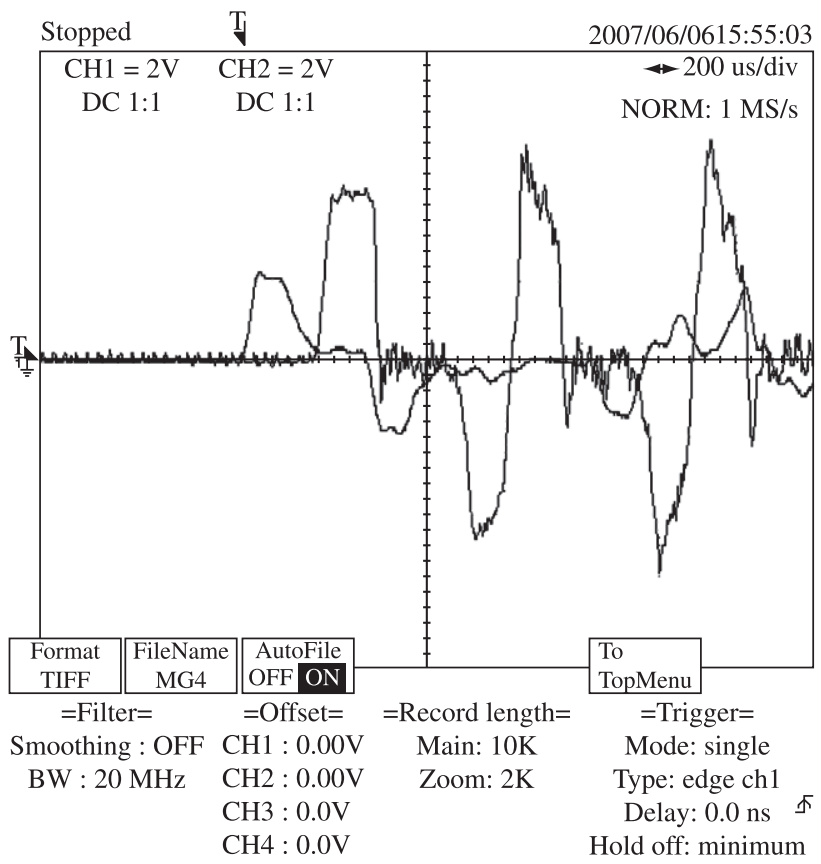

(c)

Figure 2. a) Tensile loading Hopkinson bar - schematic representation; b) picture of Hopkinson bar; and c) example of incident, reflected and transmitted waves for AZ31-B test. 


\section{Experimental Results}

The values of $R_{p 0,2}$ (proportionality limit corresponding to a permanent plastic strain of $0.2 \%$ ) and UTS from the quasi-static and dynamic uniaxial tensile tests on the magnesium and aluminium alloys are presented in Figure 3. For the AZ31B alloy, the strength values reveal a moderate but nevertheless important variation with increasing strain rate, which was expected given other results for magnesium alloys. The highest increase in strength values was observed when testing with the Hopkinson bar since the strain rates are almost one order of magnitude larger. In the aluminium alloy the change of material properties is less significant, which is expected for this type of material.

The evaluation of the elongation to fracture for the dynamic tests should be considered with care since different specimen sizes were used for quasi-static loading and at higher strain rates. Considering the limitation of comparing values obtained from samples with different gauge lengths, it was observed through SEM (Scanning Electron Microscope) of fracture surfaces that neither material suffers a degradation of ductility with increasing loading speeds. These results are presented in Figures 4 and 5, where it is noted that there is no departure from the typical morphology of ductile fracture.

\section{Constitutive Equations}

The well-known Cowper-Symonds equation is frequently used to describe material behaviour at different strain $\operatorname{rates}^{4}$ (Equation 1):

$$
\frac{\sigma_{d y n}}{\sigma_{\text {stat }}}=1+\left(\frac{\dot{\varepsilon}}{D}\right)^{\frac{1}{p}}
$$

where $D$ and $p$ are the Cowper-Symonds coefficients, $\dot{\varepsilon}$ is the strainrate, $\sigma_{d y n}$ is the dynamic stress or strength and $\sigma_{\text {stat }}$ is the quasi-static stress or strength. The coefficients obtained for Equation 1 are presented in Tables 3 and 4, along with the strain rate range of applicability and the mean squared value $\left(R^{2}\right)$ for the tested materials.

From an analysis of the results, one can conclude that it is generally possible to find the Cowper-Symonds coefficients, in some cases with excellent correlation. However, these coefficients assume different values for different reference stresses. For example, the ultimate tensile and proof stresses have different sensitivities to strain rate, which result in different coefficients in the Cowper-Symonds equation. This is an intrinsic disadvantage of using the Cowper-Symonds equation. In this case, the main risk with using Equation 1 is that, for example, coefficients determined with $R_{p 0.2}$ as the reference stress might yield an erroneous prediction for higher stress levels at larger strains. This feature can have an important effect on the numerical simulation of dynamic crushing of thin-walled tubes, as discussed in Peixinho and Pinho ${ }^{5}$.

\section{Discussion}

An analysis of the different properties presented in this study and the comparison between magnesium AZ31B and 6111-T4 aluminium alloy has shown that these materials show strong potential for further application in crashworthy automotive components and parts. However, certain features should be studied in more detail, namely the manufacturing limitations of the wrought magnesium alloys given their lower elongation to fracture. Also, this lower ductility can have an effect on the deformation modes of the crushing process of crashworthy parts, with the possibility of fracture and consequent reduced energy absorption.

The Cowper-Symonds coefficients obtained have excellent correlation with the experimental data. However, those coefficients and the associated constitutive equation need to be validated through

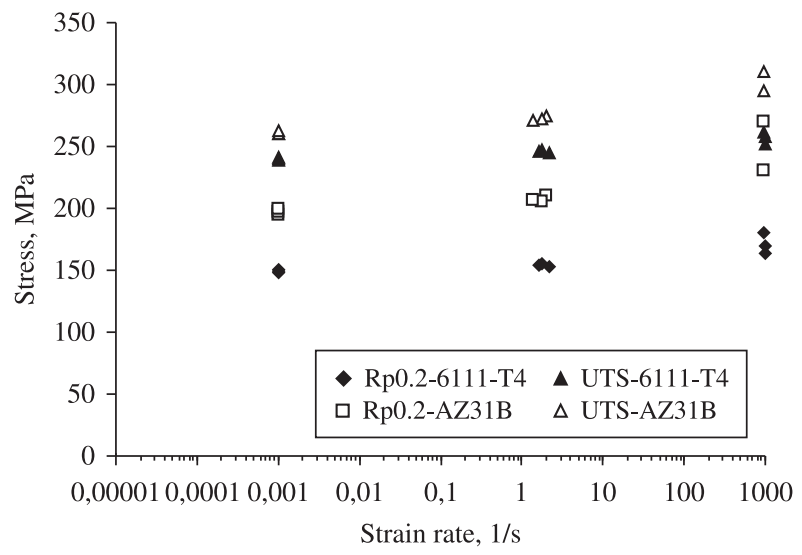

Figure 3. Variation of strength properties with strain rate for the tested alloys.

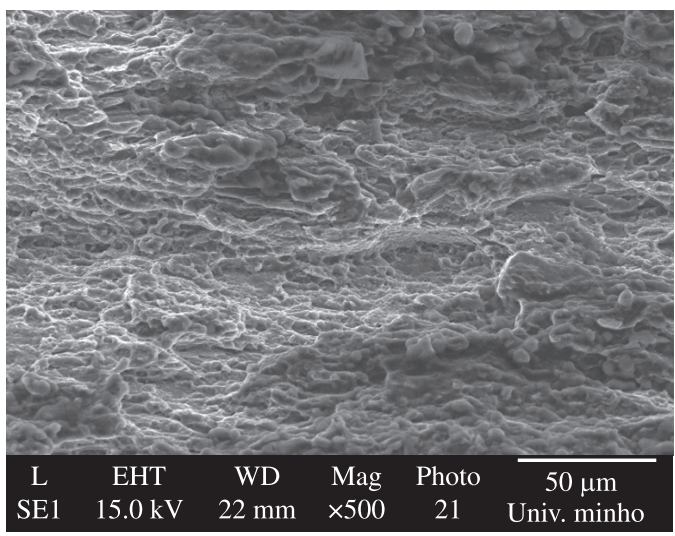

$1 \times 10^{-3} / \mathrm{s}$

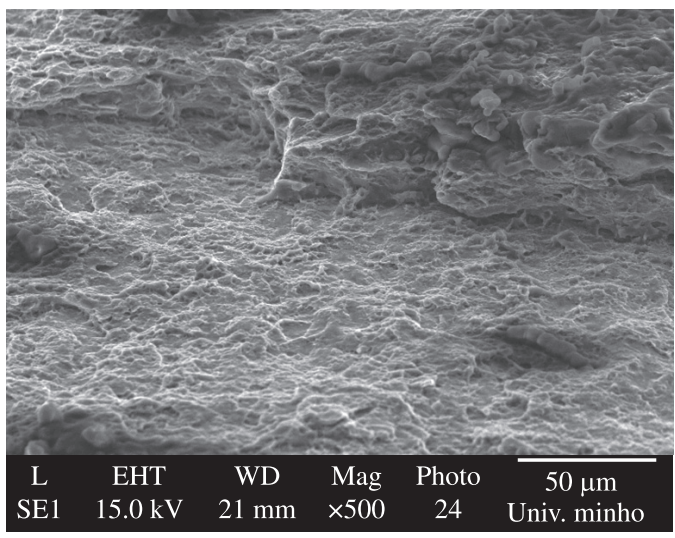

$2.25 / \mathrm{s}$

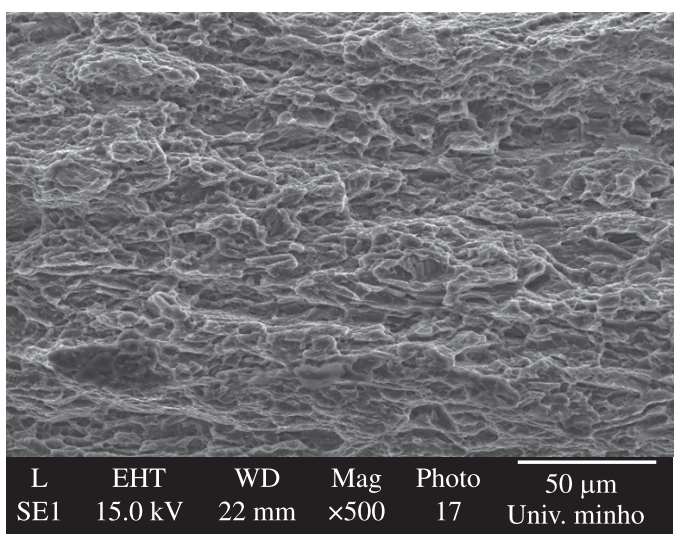

$1050 / \mathrm{s}$

Figure 4. SEM images of fracture surfaces (AZ31B) - 500×. 
Table 3. Cowper-Symonds parameters - A6111-T4.

\begin{tabular}{ccccc}
\hline & $\dot{\varepsilon}$ range $[/ \mathrm{s}]$ & $D[/ \mathrm{s}]$ & $p$ & $R^{2}$ \\
\hline$R_{p 0.2}$ & $0.001-1020$ & 415401.2 & 3.57 & 0.97 \\
UTS & $0.001-1020$ & 24106691 & 4.35 & 0.90 \\
\hline
\end{tabular}

Table 4. Cowper-Symonds parameters - AZ31B.

\begin{tabular}{lcrcc}
\hline & $\dot{\varepsilon}$ range [/s] & \multicolumn{1}{c}{$D[/ \mathrm{s}]$} & $p$ & $R^{2}$ \\
\hline$R_{p 0.2}$ & $0.001-1050$ & 24124.9 & 3.09 & 0.96 \\
UTS & $0.001-1050$ & 231191.1 & 3.56 & 0.91 \\
\hline
\end{tabular}

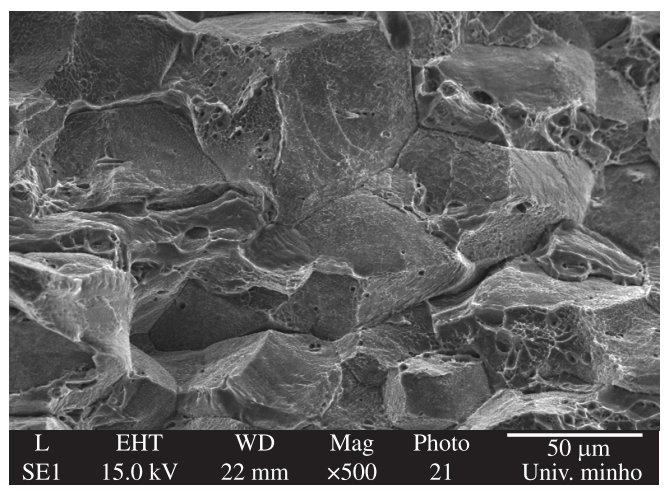

$1 \times 10^{-3} / \mathrm{s}$

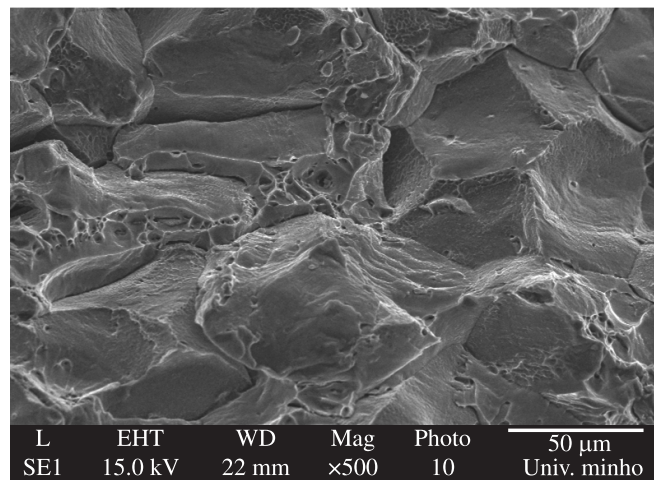

$2.25 / \mathrm{s}$

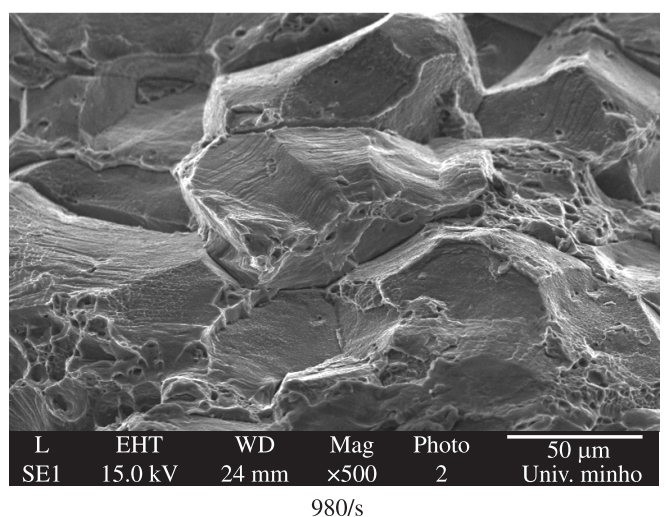

Figure 5. SEM images of fracture surfaces (6111-T4) - 500×.

the numerical simulation of the dynamic crushing of components and comparison with experimental results. The issue of variation of coefficients with reference stress should be carefully evaluated, eventually addressing the need for a more sophisticated constitutive equation.

\section{Conclusions}

This work presented results on the tensile testing of two light alloys at different strain rates in the range 0.001-1000/s. Mechanical properties were determined from tensile tests using flat sheet samples, employing two different test techniques: a servo-hydraulic machine and a tensile-loading Hopkinson bar. The test results were used to compare different mechanical properties of the tested materials. The results were also used to obtain parameters for the Cowper-Symonds constitutive equation.

The results obtained highlighted properties that qualify the materials for crashworthiness applications, namely the strain rate sensitivity. The magnesium alloy presented a higher variation of mechanical properties as strain rate increased. Neither material showed a deviation from ductile failure at the highest strain rates obtained in the Hopkinson bar.

\section{Acknowledgments}

We express our gratitude to Alcoa for supplying the tested aluminium alloy. This study was sponsored by the Portuguese Foundation for Science and Technology under project PTDC/EMEPME/65009/2006.

\section{References}

1. Miller W, Zhuang L, Bottema J, Wittebrood A, De Smet P and Haszler A. Recent developments in aluminium alloys for the automotive industry. Journal of Materials Science and Engineering. 2000; A280:37-49.

2. Bjornstad P. Presentation from Tonsberg Magnesium Group. 2006.

3. Imbert J, Winkler S, Worswick M and Golovaschenko S. Formability and damage in electromagnetically formed AA5754 and AA6111. In: Proceedings of the first international conference on high speed forming; 2004; Dortmund, Germany. p. 202-210.

4. Jones N. Structural Impact. Cambridge University Press; 1997.

5. Peixinho $\mathrm{N}$ and Pinho A. Study of viscoplasticity models for the impact behaviour of high-strength steels. Journal of Computational and Nonlinear Dynamics. 2007; 2:114-123.

6. Tanaka $\mathrm{K}$ and Nojima T. Strain rate change tests of aluminium alloys under high strain rate. In: Proceedings of the 19th Japan congress on materials research; 1975; Tokyo, Japan. p. 48-51.

7. Lindholm U, Bessey R and Smith G. Effect of strain rate on yield strength, tensile strength and elongation of three aluminium alloys. Materials Science and Engineering A. 1971; 6(1):119-133.

8. Oosterkamp L, Djapic, Ivankovic A and Venizelos G. High strain rate properties of selected aluminium alloys. Journal of Materials Science and Engineering. 2000; A278:225-235.

9. Li L, Zhou J and Duszczyk J. Determination of a constitutive relationship for AZ31B magnesium alloy and validation through comparison between simulated and real extrusion. Journal of Materials Processing Technology. 2006; 172:372-380.

10. Valle $\mathrm{J}$ and Ruano $\mathrm{O}$. Influence of the grain size on the strain rate sensitivity in an $\mathrm{Mg}-\mathrm{Al}-\mathrm{Zn}$ alloy at moderate temperatures. Scripta Materialia. 2006; 55:775-778.

11. Peixinho $\mathrm{N}$ and Pinho A. Dent resistance of aluminium and magnesium alloys, Journal of Automobile Engineering. 2006; 220:1191-1198.

12. Kang W, Cho Huh H and Chung D. Modified Johnson-Cook Model for Vehicle Body Crashworthiness Simulation. International Journal of Vehicle Design. 1999; 21(4/5):424-435. 